\title{
Bulk and Nanoscale Polypeptide Based Polyelectrolyte Complexes
}

Amanda B. Marciel ${ }^{1}$, Eun Ji Chung1,2, Blair K. Brettmann ${ }^{1}$, and Lorraine Leon ${ }^{1 *}$

1) Institute for Molecular Engineering, University of Chicago, Chicago, IL 60637

2) Department of Biomedical Engineering, University of Southern California, Los Angeles, CA 90089

*Corresponding Author: lorraineleon@uchicago.edu

\section{Abstract}

Polyelectrolyte complexes (PECs) formed using polypeptides have great potential for developing new self-assembled materials, in particular for the development of drug and gene delivery vehicles. This review discusses the latest advancements in PECs formed using polypeptides as the polyanion and/or the polycation in both polyelectrolyte complexes that form bulk materials and block copolymer complexes that form nanoscale assemblies such as PEC micelles and other self-assembled structures. We highlight the importance of secondary structure formation between homogeneous polypeptide complexes, which, unlike PECs formed using other polymers, introduces additional intermolecular interactions in the form of hydrogen bonding, which may influence precipitation over coacervation. However, we still include heterogeneous complexes consisting of polypeptides and other polymers such as nucleic acids, sugars, and other synthetic polyelectrolytes. Special attention is given to complexes formed using nucleic acids as polyanions and polypeptides as polycations and their potential for delivery applications. Keywords: Polypeptides, Polyelectrolytes, Coacervates, Self-Assembly, Micelle 


\section{Introduction}

Polyelectrolyte complexes (PECs) are formed by mixing oppositely charged polyelectrolytes, which can occur by creating a composite material that macrophase or microphase separates from solution[1,2], adsorbs sequentially on a surface as is the case in electrostatic layer-by-layer assembly $[3,4]$, or on the surface of "hairy colloids" containing grafted polyelectrolytes[5], the former of which is the main focus of this review. Although related phenomena, this definition does not include the mixing of polymers with oppositely charged colloids[6,7] or surfactants[8] since both components are not polymeric. Polyelectrolyte complexation is entropically driven by the release of counterions initially confined to the double layer, which occurs when the oppositely charged polymers come together. $[9,10]$ The nature of these phase separated complexes can be solid, in the form of a precipitate, or liquid, in the form of a coacervate.[1] Precipitates have found limited utility as functional materials without special processing, such as the addition of salt.[11] In contrast, the coacervate phase contains very high water content[12] and has very low interfacial tension with water. [13-15] These properties make coacervates important in a variety of different applications and scenarios. For example, coacervates are used to encapsulate flavors and additives in the food industry[16] , as underwater adhesives for marine organisms[17], have been implicated in origin 
of life scenarios[18-20], used as electronic ink[21], in direct ink writing[22,23], and as potential drug delivery vehicles[2,24]. Polyelectrolyte complexation can occur as either a macroscopic phase separation that form bulk materials or a microphase separation when block copolymers are used, enabling the formation of nanoscale PECs. $[2,9,25]$

This review focuses on PECs formed as bulk and nanoscale materials using polypeptide based polyelectrolytes. Numerous studies have focused on using complex biopolymers and proteins to study PEC formation, which have the benefit of being biocompatible, but are structurally complex. [26] Polypeptides provide an interesting alternative to these complex biopolymers, due to their inherent biocompatibility (excluding high concentrations and molecular weights of polycationic polypeptides[27]) and simple polymeric structure, which can allow for a more systematic investigation into PEC phenomena. Moreover, polypeptides can form secondary structure motifs that can mimic protein behavior and introduce additional intermolecular forces such as hydrogen bonding that influence the stability and phase behavior of PECs. [28,29]

Polypeptide based materials can be synthesized using standard fluorenylmethyloxycarbonyl chloride (FMOC) based solid phase synthesis. [30,31] This technique grows the polypeptide chain on a porous polystyrene support one amino acid at a time, allowing for the polypeptides to have specific charge distributions and sequences that are not accessible using other polymerization methods. [30,31] In addition, the polypeptides prepared using solid phase synthesis have relatively low polydispersities compared to other polymerization methods, but 
relatively low molecular weights of less than 100 amino acids. To create block copolymer polypeptides, polymers containing carboxylic acid functional groups can be conjugated to the polypeptide chains.[32] For higher molecular weight polypeptides, $\mathrm{N}$-carboxyanhydride (NCA) ring opening polymerization is used, which can create polypeptide chains larger than 200 amino acids in length. This technique facilitates large scale synthesis of polypeptides in gram quantities and has benefited from recent advancements in novel catalysts and better NCA purification.[33] Block copolymers containing polypeptide segments can be synthesized using this method by initiating the polymerization from a polymer containing a terminal amine functionality.[33]

\section{Bulk Polyelectrolyte Complexes}

Mixing oppositely charged polyelectrolytes results in the formation of bulk PECs. Liquid complexes, or coacervates are characterized by the formation of micron sized spherical droplets that can coalesce and form a distinct phase.[34] On the other hand, precipitates form irregularly shaped amorphous solids that can be re-suspended after centrifugation.[12] Images of both types of complexes can be observed in Figure 1. This section discusses the formation of bulk PECs formed using a polypeptide as the polycation, polyanion, or both.

\section{Homogeneous Polypeptide Complexes}

Homogeneous PECs are formed when both the polyanion and polycation are polypeptides. An extensive study on PECs using polylysine (pLys) and polyglutamic acid (pGlu) investigated the effects of pH, temperature, molecular weight, polyanion/polycation mixing ratio and total polymer concentration on the PEC 
formation phase diagram, concluding that polypeptide based complexes have similar tendencies to other PEC systems.[12] A related study probed the thermodynamic driving forces of polypeptide complexation using isothermal titration calorimetry and found that the process was entropically driven and endothermic.[10] The interfacial energy of homogeneous polypeptide coacervates has been measured to be extremely low $(<1 \mathrm{mN} / \mathrm{m})$ using a surface forces apparatus[14], which is also comparable to other coacervate systems.[13] Choosing polypeptides as polyions allows for very precise control over charge spacing and polydispersity, especially when synthesized using solid phase methods. An advanced Monte Carlo simulation with a replica exchange algorithm combined with experimental techniques allowed for the investigation of charge distribution and charge matching in three model systems that use glycine residues as spacers in both lysine and glutamic acid based polyelectrolytes: $(\mathrm{EGEG})_{5} /(\mathrm{KGKG})_{5},(\mathrm{EEGG})_{5} /(\mathrm{KKGG})_{5}$, and $(\mathrm{EEGG})_{5} /(\mathrm{KGKG})_{5}$.[35] Zhao and colleagues conclude that the size, density, and packing of the complexes can be tuned with the distribution of neutral spacers, such that the (EGEG) $)_{5} /(\mathrm{KGKG})_{5}$ complexes, where the charged residues alternate 1-to-1 with the neutral residues, have the highest density and molecular weight found using laser light scattering, and this is in agreement with the largest aggregation number found using the simulations.[35] Interestingly, the density, molecular weight, and aggregation number of the mismatched complex, $(\mathrm{EEGG})_{5} /(\mathrm{KGKG})_{5}$, was higher than both of the matched cases, indicating that charge matching also influences the complexation phenomena.[35] 
Polypeptide based polyelectrolytes can be synthesized in gram scale quantities using NCA ring opening polymerization methods.[33] These methods do not allow for precise control over the sequence, but can still form molecules of interest, including incorporating amino acids in the same proportions as those found in sand castle worm proteins, which form coacervates with exceptional adhesive properties.[17] Zhang et al. created a cationic polypeptide consisting of lysine, di-hydroxyphenylalanine (DOPA), glycine, and tyrosine and an anionic polypeptide consisting of phosphoserine, serine, and tyrosine; both in gram scale quantities. Mixing these polypeptides together created liquid coacervate complexes with low surface tension, shear-thinning behavior and a viscoelastic response, providing a robust synthetic method for creating biomimetic materials.[36]

One of the unique properties of polypeptide-based complexes involves the ability for secondary structure formation in the form of $\beta$-sheets and $\alpha$ - helices. Recently, it has been shown that homochiral polypeptide based complexes form $\beta$ sheet structures that facilitate the formation of solid complexes, while racemic polypeptides inhibit $\beta$-sheet formation due to steric hindrance and create liquid complexes, illustrating mechanisms to alter PEC phase behavior using secondary structure formation.[28,37] This work included both experimental results and molecular dynamics simulations that illustrated the same conformations.[28,37] Images of the complexes formed can be seen in Figure 1. Using synthetic techniques that were designed to increase the spacing between the charged group and the polypeptide backbone allowed the creation of polypeptides that were simultaneously charged and $\alpha$-helical.[38] These helical polyelectrolytes formed 
liquid coacervate phases when mixed with polyglutamic acid (pGlu), regardless of the polyanion chirality due to the reduced intermolecular hydrogen bonding arising from the intramolecular $\alpha$-helical structure.[29] Interestingly, the critical salt concentration of the coacervates containing helical polypeptides is extremely high compared to regular ionic polypeptide complexes, likely due to the increased charge density of the helical polyelectrolyte. [29]

\section{Heterogeneous Polypeptide Complexes}

Heterogeneous polypeptide complexes may be formed by mixing a polypeptide with synthetic polymers or biopolymers. However, most heterogeneous polypeptide complexes of interest involve biopolymers, specifically nucleic acids.

\section{Polypeptide-Nucleic Acid Complexes}

Recently, PECs have gained interest as potential non-viral DNA, RNA and oligonucleotide delivery vectors for gene therapy technologies.[39] Nucleic acid drugs, unlike low molecular weight hydrophobic drugs, cannot freely pass through the cell membrane due to their highly charged backbones and large molecular weights, which ultimately inhibits delivery to target sites and decreases drug efficacy.[40] To overcome these challenges, initial strategies utilized viral delivery vectors, which intrinsically have the ability to enter specific cells, escape endosomal compartments, and highjack cellular machinery to efficiently transport nucleic acid cargo.[41] However, viral vectors can induce an adverse immune response due to remaining viral capsid surface proteins.[40] In addition, from a technical perspective, viral vectors are difficult to genetically engineer, challenging to produce 
at large scale and have the ability to revert back to their wild-type viruses. For these reasons, several non-viral alternative strategies have been developed based on either physical methods (e.g., electroporation, gene gun, sonication, DNA injection, etc.) or supramolecular assembly strategies (e.g., PECs, liposomes, micelles, etc.).[40]

The most prominent non-viral delivery methods utilize cationic lipids and polymers to form PECs with negatively charged nucleic acids. However, it is often unclear if these PECs form precipitate or coacervate structures. These PECs, commonly referred to as polyplexes, are designed to mimic viral delivery vectors by first compacting and then targeting nucleic acids to intended tissues and cells. [4144]This alternative delivery strategy has gained popularity because of its low immune response, facile production of large quantities, and temporary gene expression without introduction into the host chromosome.[40] However, achieving efficient nucleic acid delivery is difficult due to several extra- and intracellular barriers. [40,45] The following polyplex design parameters have shown to be critical for successful nucleic acid delivery, (i) stability to protect nucleic acids from enzymatic degradation, (ii) reduced non-specific interactions to avoid aggregation and subsequent accumulation in off-target organs, (iii) targeting specificity into desired tissue and cells, (iv) endosomal disruption to escape into the cytoplasm, ( $v$ ) release of the nucleic acid cargo in the cytoplasm or transport into the nucleus for transcription, and (vi) integration of solely non-toxic components. [40,46]). In light of these design parameters, cationic polymer-based nucleic acid delivery vectors are 
the most promising due to their versatile synthesis, which allows incorporation of a wide array of additional functionalities.

In particular, peptide based cationic polymers have been extensively investigated as non-viral nucleic acid delivery vectors due to their biocompatibility, low toxicity, and weak activation of the complement system.[46] In addition, synthetic parameters like chemical composition, chain length, charge density and topology can be easily tuned to modulate complexation kinetics and ultimately the final polyplex structure.[47] The physiochemical properties of peptide based polyplexes (e.g., size, surface charge, interaction strength, colloidal stability) may also be altered via preparation conditions, including ionic strength, $\mathrm{pH}$, concentration, solvent quality, and mixing order.[47] To overcome the aforementioned extra- and intracellular barriers, specific classes of peptides are integrated into polyplex delivery vectors, including nucleic acid complexation peptides (high lysine and arginine content), cell-penetrating peptides (CPPs), membrane perturbing peptides and peptides containing nuclear localization sequences (NLSs).[46] However, the physiochemical properties of polyplexes, including phase behavior, and their effects on nucleic acid delivery are still not well understood. Therefore, there is a strong need to investigate the underlying mechanisms that drive polyplex formation and develop an understanding of their delivery behavior in vitro and in vivo.

Initially, pLys was utilized as a model polymer to investigate plasmid DNA (pDNA) complexation.[40,48] pLys is positively charged at neutral $\mathrm{pH}\left(\mathrm{p} K_{\mathrm{a}}\right.$ of $\varepsilon$ amine is around 10.8) and has the ability to electrostatically interact with the 
negatively charged phosphate backbone of nucleic acids, thus forming polyplexes. Stability of pLys/pDNA polyplexes is dependent on pLys molecular weight, the amine-to-phosphate charge ratio (N:P), and the preparation conditions.[40] Generally, it has been shown that pLys/pDNA polyplexes are unstable if pLys has a molecular weight below $3000 \mathrm{Da}$ and if the N:P ratio is unity - due to a loss of electrostatic repulsion between polyplexes resulting in aggregation.[40,49] However, determining polyplex stability a priori remains challenging. In 2001, Lai et al., investigated the underlying kinetic processes of pLys/pDNA complexation to determine the influence on polyplex size and dispersion. Using time-resolved multiangle laser light scattering, they showed that at high and low mass ratios both polyplex molar mass and geometric size remained constant over several hours, indicating that initial complexation is rapid. However, at intermediate mass ratios, close to an N:P ratio of 1, polyplex molar mass and geometric size increased over several hours, resulting in polyplexes containing multiple pDNA molecules. Therefore, the N:P ratio has a substantial role in polyplex size, dispersion and pDNA loading.[48] To further elucidate polyplex complexation mechanisms, Mann et al. utilized atomic force microscopy (AFM) to investigate the combinatorial effects of the N:P ratio, pLys molecular weight and pDNA concentration. They found that at low N:P ratios complexation is monomolecular at low pDNA concentration and multimolecular at high pDNA concentration. In addition, they determined that low molecular weight pLys showed the most efficient complexation with low pDNA concentration, whereas the higher molecular weight pLys showed the most efficient complexation at high pDNA concentration.[45] Despite the ability of pLys to 
effectively complex nucleic acids, it exhibits relatively high cytotoxicity, the tendency to form aggregates and poor endosomal escape, thus decreasing drug efficacy. [40,49]

Several pLys derivatives have been developed to increase delivery efficiency. Examples include incorporation of imidazole groups to enhance endosomal escape, disulfide bonds and acid-labile groups to aid in triggered release of nucleic acids, cross-linking and coating capabilities to increase stability and decrease overall surface charge, as well as specific peptide sequences for targeting and endosomal escape. [41,49] To increase the diversity of possible stimuli responsive gene delivery vectors Oupický et al. developed novel temperature-dependent pLyspoly( $N$-isopropylacrylamide) (PNIPAM) graft copolymers.[50] Introduction of PNIPAM simultaneously caused significant changes in polyplex surface charge and structural density. Below the transition temperature $\left(32^{\circ} \mathrm{C}\right)$ the structural density and surface charge decreased with increasing PNIPAM, whereas above the transition temperature the structural density and surface charge increased with increasing PNIPAM.[50]

In addition, polymer architecture is an essential parameter that can be utilized to tune nucleic acid delivery efficiency. For example, dendritic pLys exhibited reduced cytotoxicity as compared to linear pLys.[49] A recent investigation by Parelkar et al. emphasized the importance of polymer architecture by developing several poly(cyclooctene-graft-oligolysine) comb polymers with different pendent length oligolysines, as well as graft copolymers that integrated the PKKKRKV NLS heptapeptide for nuclear localization, which both displayed superior 
nucleic acid delivery efficiency compared to linear pLys. Depending on the length of oligolysine grafts, polyplexes exhibited good stability in fetal bovine serum (FBS) and release of pDNA upon heparin treatment. In the DNase I treatment, polyplexes showed decomplexation with little-to-no evidence of pDNA degradation, which indicates that these polyplexes protected the pDNA payload but are loosely arranged. The authors postulated that delivery efficiency is related to polyplex morphology and pDNA binding strength.[42] An earlier approach using comb shaped polymers involved the development of polyhistidine(pHis)-graft-pLys comb polymer, which was used to complex pDNA. The pHis-g-pLys complex improved the transfection of pDNA in 293T cells compared to pLys likely due to enhanced endosomal release via incorporation of the $\mathrm{pH}$ sensitive imidazole groups.[51] Comb polymers containing pLys and polyethylene glycol (PEG) have also successfully demonstrated reduction in the cytotoxicity of pLys, while retaining transfection efficiency.[52]

It is difficult to design a single polymer capable of nucleic acid complexation with efficient extra- and intracellular delivery properties. Therefore, several multicomponent non-viral delivery vectors have been developed. Soliman et al. designed a viral-mimetic core-shell polyplex architecture with a reducible cationic polypeptide core and a hydrophilic polymer coating that contains acid-labile linkages as well as cell targeting functionality.[41] Utilizing an N:P ratio of 5, reducible polycations with varying histidine and lysine sequences (linked via cysteine termini) were complexed with pDNA. Following initial complexation, noncleavable bis- and tetra(oxirane) PEG shield polymers and acid-labile bis- and 
tetra(oxirane)-acetal terminated PEG shield polymers were reacted with the preexisting polyplexes. Via gel retardation assays and AFM, it was confirmed that pDNA was protected at neutral $\mathrm{pH}$ and released at $\mathrm{pH}$ 5.6, while the core polyplex released pDNA under reducing conditions. In a similar strategy, Sanjoh et al. developed a ternary polyplex delivery vector with dual environmental sensitivity as illustrated in Figure 2.[53] Initially, a cross-linked binary polyplex is formed via pDNA and thiolated pLys. The core polyplex is then coated with a membrane-active polyanion, PEG-b-poly(aspartamide(DET-Aco)) that degrades in the acidic endosomal environment and facilitates escape by disrupting the membrane. In the cytoplasm, the pDNA is released post cleavage of the pLys disulfide bonds and the decomplexed shield polymer is deprotonated to its less cytotoxic state.[53]

Although the majority of investigations on PECs as non-viral delivery vectors have focused on delivery of pDNA, there is growing interest in non-viral delivery of low-molecular weight oligonucleotides, in particular siRNA (short double-stranded RNA), as well as antisense oligonucleotides (short single-stranded DNA) due to their potential in therapeutic applications. However, there has been little success in developing PECs for oligonucleotide delivery because complexation typically results in formation of large and unstable polyplexes under physiological conditions. $[54,55]$ Structural differences between pDNA and oligonucleotides, like flexibility (e.g., persistence length ssDNA $l_{\mathrm{p}} \approx .6 \mathrm{~nm}$ versus dsDNA $l_{\mathrm{p}} \approx 53 \mathrm{~nm}[56]$ ) and charge density (e.g., ssDNA versus dsDNA) may be the main reason for differences in complexation behavior. Stevenson et al., showed that polyplexes containing siRNA exhibited enhanced complexation behavior as cationic polymer molecular weight 
decreased, contrary to pDNA.[57] Poor complexation stability has also been addressed via siRNA chemical modifications, including PEG-siRNA, sticky-siRNA, poly-siRNA and multimerized siRNA.[55] Takemoto et al. developed an siRNAgrafted poly(aspartic acid) via disulfide linkages, which allows for cleavage under reductive environments. The increased charge number and density aided in stable polyplex formation with a poly(aspartamide) modified for endosomal escape.[55] Polyplex stability was evaluated in FBS, followed by a counter polyaninon exchange with heparin and showed that the grafting of siRNA is critical for the increased stability of the polyplex.

Zheng et al. systematically investigated the effects of nucleic acid length and hybridization state on polyplex formation. In this study, the complexation behavior of three different types of DNA (2000 bp dsDNA, 21 bp ds-oligo and $21 \mathrm{nt}$ ss-oligo) with pLys was investigated using laser light scattering (LLS) and AFM.[58] They found that unlike long dsDNA that forms stable loose complexes with pLys, the oligonucleotides initially form more compact polyplexes that are unstable and disassociate over time.[58] In a follow up study, they investigated the long-term kinetics of polyplex formation between two different lengths of dsDNA (2000 bp DNA or 21 bp DNA) and pLys. Using LLS, complexation exhibited three stages: (i) formation of the preliminary complex, (ii) further aggregation and (iii) precipitation. They postulate that short chains have a lower barrier to minimize free energy and can reorganize inside and amongst polyplexes, which results in further/faster aggregation or precipitation. 
Polypeptide Complexes with Proteins, Polysaccharides and Other Synthetic Molecules

In addition to polypeptide-nucleic acid complexes, heterogeneous complexes can include polypeptides complexed with biological components such as proteins or polysaccharides..[59-62] Recently, Black and colleagues developed a simple and versatile method for encapsulating proteins via complex coacervation between pLys and racemic pGlu.[59] To achieve this, a model protein system, bovine serum albumin (BSA) was first mixed with pLys to form an intermediate complex with a net negative charge at neutral pH. Subsequently, racemic pGlu, was added and the protein-containing complex coacervates were formed (Figure 3). Maximum loading was determined to be one BSA per pLys/pGlu pair. The secondary structure of the encapsulated protein remained the same within the complex coacervate system and the protein was released under conditions of decreasing $\mathrm{pH}$.

Complexation of pLys with polysaccharides have also been proposed for drug delivery applications. $[60,61,63]$ Girod et al. report on the PEC formation between iota - (l)carrageenan and pLys with equal charge stoichiometry.[60] Carrageenans are polydisperse linear sulfated galactan sugar molecules extracted from various red algae species that have been extensively used in the food and pharmaceutical industry as gelling agents and for microcapsule formation in association with other polyelectrolytes.[64] Other common polysaccharides complexed with polypeptides include hyaluronic acid (HA) and chitosan. [61,65]

\section{Polypeptide Micellar Polyelectrolyte Complexes}


Both coacervates and precipitates can be confined to the nanoscale using diblock or triblock copolymers consisting of neutral polymers tethered to polycations or polyanions. The copolymer can serve as the polyanion, polycation, or both to ensure nanoscale stabilization of the PEC. PEC micelles are made up of a PEC core surrounded by a neutral corona and have also been called polyion complex (PIC) micelles[66], complex coacervate core micelles[2], and interpolyelectrolyte complex micelles.[25] This section describes PEC micelles formed using charged polypeptides as core forming segments.

\section{Homogeneous Micellar Complexes}

Similar to bulk PECs, homogeneous micellar complexes are formed using polypeptides as both the polyanion and polycation. Pioneering work forming polypeptide based PEC micelles (termed PIC micelles) originated in Kataoka's group using polyethylene glycol (PEG)-b-polylysine (PEG-pLys) and PEG-poly $(\alpha, \beta$-aspartic acid) (PEG-pAsp) that were synthesized using ring opening polymerization of NCAs.[66] Using diblock copolymers as both anions and cations and mixing them at an equal charge molar ratio created micelles that were monodisperse and spherical, which was confirmed using light scattering techniques.[66] Further studies in the Kataoka group examined the mixing of diblock copolymers with different lengths of polyelectrolyte chains using a combination of gel filtration chromatography and light scattering. They showed that micelle formation only occurred when both the PEG-pLys and PEG-pAsp had similar degrees of polymerization for their charged components.[67] Interestingly, the micelles exhibit this chain length recognition phenomena and select the polyelectrolyte with a matching chain length even in 
polymer mixtures consisting of multiple chain lengths, highlighting an interesting separation mechanism.[67] However, micelles formed by mixing PEG-pAsp and pLys were able to form regardless of polyelectrolyte length mismatch, indicating a larger degree of chain rearrangement. [68,69] This difference between double diblock micelles and diblock/homopolymer micelles suggests that there must be a sharp interface between the core and corona forming segments.[69] By mixing PEGpLys with varying mixtures of homopolymers and diblock copolymers (pAsp and PEG-pAsp), different structures such as cylindrical micelles, connected cylindrical micelles, and lamellae were formed.[70] The structures deviated from sphericity as the PEG fraction in the system decreased and could be changed with increasing temperature due to the decreasing volume occupied by the PEG segment as seen in Figure 4.[70]

Secondary structure also plays a role in micellar polypeptide PEC formation. When homochiral PEG-pLys was mixed with pGlu, circular dichroism revealed that the micellar core contained $\beta$-sheet content, which is in contrast to PEG-pLys mixed with racemic pGlu, where a random coil structure is obtained.[28] The micelles containing $\beta$-sheet content, termed solid core micelles, had slower kinetics of formation and higher polydispersity, consistent with their inability to rearrange into equilibrium structures due to the hydrogen bonding of the chains.[28] This study was able to highlight differences in solid and liquid core micelle behavior by direct comparison to bulk systems in which coacervate and precipitate phases are more easily identified.[28]Similar reports were also made using PEG-pGlu and homochiral or racemic pLys, where the homochiral pairs had $\beta$-sheet content that depended on 
concentration and ionic strength.[71] Micelles with $\alpha$-helical content have also been reported using PEG- poly(g-3-(4-(guanidinomethyl)-1H-1,2,3- triazol-1-yl)propyl-1glutamate (PPLGPG), the diblock copolymer version of the helical and charged polypeptide described in the bulk complexes section. [29] Considerations of how secondary structure in the micellar core relates to micelle stability have yet to be explored, but may present a unique opportunity for encapsulation of charged therapeutics.

In addition to understanding the formation and properties of micellar polypeptide PECs, there is increasing interest in developing stimuli-responsive micelles using a variety of molecular design approaches. By creating a diblock copolymer with thiolated lysine units using a heterobifunctional crosslinking agent, it was possible to crosslink the micelle core using disulfide bonds.[72] The crosslinked micelles were more stable to increasing salt concentrations than their non-crosslinked counterparts and could be reduced by addition of dithiothreitol, indicating a mechanism for intracellular destabilization.[72] Other strategies for responsive assemblies have focused on temperature using thermosensitive poly-(2isopropyl-2-oxazoline) (PiPrOx), which experiences cloud point behavior upon increasing temperature. [73] Both PiPrOx-pLys and PiPrOx-pAsp were synthesized and mixed to form micellar complexes containing a polypeptide core and PiPrOx corona. Interestingly, the micelles had lower cloud point temperatures compared to the individual polymers, likely due to the increased concentration of the PiPrOx chains on the micelle corona compared to their concentration in solution.[73] Other mechanisms for micelle responsiveness have relied on $\mathrm{pH}$ and sugar presence by 
introducing boronic acid-catechol interactions into PEG-polypeptides. PEGpoly(Glu-co-glutamicamidophenylboronic acid(GLuPBA) and PEG-b-pLys-co- $\varepsilon-3,4-$ dihydroxyphenylcarboxyl-L-lysine(LysCA) were synthesized by modification of the glutamic acid residues and lysine residues after NCA ring opening polymerization of pGlu and pLys. [74] Mixing of the polymers formed micelles that were more stable than their strictly polypeptide counterparts under physiological conditions and in serum due to the crosslinking of the cores, but were also sensitive to changes in $\mathrm{pH}$ and fructose concentrations. [74] Moreover, proteins were encapsulated in the micelles and released into cancer cells, illustrating potential of using these techniques for protein delivery.[74]

\section{Heterogeneous Micellar Complexes}

Similar to bulk heterogeneous complexes, micellar complexes can also be formed using polypeptides and other polymers such as nucleic acids, proteins, and other synthetic polymers. Micellar complexes containing nucleic acids are often referred to as polyplex micelles. In this section, the polypeptide molecule can be the block copolymer (anion or cation), or also the homopolymer.

\section{Micellar Polypeptide/Nucleic Acid Complexes}

Initial investigations aimed to increase the delivery efficiency of bulk

polyplexes by utilizing A-B block copolymers that contained hydrophilic PEG blocks to neutralize the surface charge and prevent non-specific interactions and cationic pLys blocks to complex with pDNA. [75,76] PEG-pLys/pDNA complexes form coreshell structures called polyplex micelles that exhibit greater delivery efficiency than bulk pLys/pDNA polyplexes. [76,77] Similar to bulk polyplexes, it is often unclear if 
the core of polyplex micelles is made up of coacervate or precipitate. Katayose and Kataoka investigated the physiochemical properties of PEG-pLys/pDNA polyplex micelles and showed an approximate diameter of $50 \mathrm{~nm}$ with small absolute values of $\zeta$ potential consistent with a core-shell structure.[77] In addition, the PEGpLys/pDNA polyplex micelles remain soluble at an N:P ratio of unity, unlike bulk pLys/pDNA polyplexes that readily aggregate and precipitate. [77]

The neutral surface charge, solubility at N:P of unity, and nuclease-resistance of PEG-pLys/pDNA polyplex micelles establishes them as attractive candidates for in vivo nucleic acid delivery.[78] Harada-Shiba et al. tested a variety of PEGpLys/pDNA polyplex micelle compositions for their stability in the blood stream and delivery efficiencies.[79] Using an in vivo turnover study, the highest stability and transfection efficiencies were exhibited at high N:P ratios and pLys length, thus demonstrating that the polyplex micelle diameter and pDNA binding strength are important factors in determining stability. $[79,80]$ Recent investigations have focused on the PEG-pLys/pDNA micelle polyplex structure, which contains a single pDNA packaged within a polyplex micelle core into a rod structure. [81,82] Interestingly, the structure adopted by the PEG chains plays a role in the delivery efficiency, where more crowded PEG blocks result in longer blood circulation times.[82] However, successful transfection of PEG-pLys/pDNA polyplex micellar systems relies upon addition of cholorquine to aid in endosomal escape.

Therefore, several studies have incorporated additional functionalities into polyplex micelles to overcome extra- and intracellular delivery barriers. Putnam et al. designed PEG-polyhistidine block and graft copolymers that both complexed with 
pDNA to form polyplex micelles. In this case, the stability of the polyplex micelles was influenced by the pH, PEG content and block copolymer architecture; however, delivery efficiencies were similar to bulk pLys/pDNA polyplexes.[75] Another approach used PEG-b-pLys-g-pHis to complex with pDNA in order to facilitate endosomal escape. By comparing different compositions, Bikram et al. found that 16 mol\% histidine had the highest transfection efficiency with the lowest toxcicity.[83] PEG-pLys conjugated via a cysteine residue exhibited comparable transfection efficiencies to PEI/pDNA polyplexes in Balb/c mice treated intranasally, but without the inflammation response.[84] In a complementary study, PEG-CysteinepLys/pDNA polyplex micelles were modified with polyhistidine to aid in endosomal escape, resulting in increased in vitro and in vivo delivery efficiency as compared to naked pDNA and PEG-Cysteine-pLys/pDNA polyplex micelles. However, this is most likely a cumulative affect of increased cellular uptake and altered intracellular trafficking.[85]

Recently, a polyplex micelle system was developed containing PEG- $b$ poly(Asp(DET)) and pDNA that exhibited negligible cytotoxicity and high transfection efficiencies.[86] The PEG-b-poly(Asp(DET)) is a PEG-polyaspartamide derivative with a 1,2-Diaminoethane group on each side chain that destabilizes the endosomal membrane at acidic $\mathrm{pH}$ via formation of a diprotonated form. [86,87] The resulting structure of PEG- $b$-poly(Asp(DET))/pDNA polyplex micelles can be tuned via $\mathrm{NaCl}$ concentration, where at $0 \mathrm{mM}$ polyplex micelles are rod-like and at $600 \mathrm{mM}$ they display toroidal structures.[88] Interestingly, for polyplex micelles with a rod or toroid morphology, changing the $\mathrm{NaCl}$ concentration results in a 
transition to toroid or string, respectively, but polyplex micelles with a string morphology do not undergo any transition and toroid morphologies do not transition to rod. The authors suggest that this unidirectional structure change reflects the thermodynamic stability of the structures.[88]

In 2014, the PEG- $b$-poly(Asp(DET))/pDNA polyplex micelle system was adapted for photochemical internalization (PCI), which utilizes light to overcome extra- and intracellular delivery barriers.[89] Initially, the triblock copolymer PEGpAsp(DET)-pLys is complexed with pDNA forming a three-layered polyplex micelle structure consisting of a pLys/pDNA core, an intermediate pAsp(DET) layer and outer PEG corona, which was verified via ${ }^{1} \mathrm{H}$ NMR. Next, the photosensitizer dendrimer phthalocyanine (DPc) which consists of 32 carboxyl groups, electrostatically interacts with the intermediate pAsp(DET) layer to create the final multifunctional DPc-loaded ternary polyplex micelle (DPc-TPM). In vitro studies showed that upon cellular uptake, DPc is released due to protonation of the carboxyl groups which enhances hydrophobic interaction with the endosomal membrane. Upon photoirradiation, the DPc disrupts the membrane via formation of reactive oxygen species (ROS) and subsequently the three-layered polyplex micelle can escape into the cytoplasm. The DPc-TPM exhibited significantly higher gene expression compared to the polyplex micelle without DPc/Asp(DET). In addition, the authors demonstrated the first successful in vivo demonstration of PCI mediated nucleic acid delivery using DPc-TPM.[89]

Polyplex micelles can be formed using other oligonucleotides (ODNs) such as siRNA, mRNA, or antisense DNA using polypeptide based diblock copolymers. [90- 
96]. However, unlike polyplex micelles, the charged block of the diblock copolymer can also be the oligonucleotide.[97-101] In the most basic system, Kataoka et al. utilized a PEG-pLys block copolymer as the counterpart to an antisense ODN, preparing spherical micelles with a small size $(\sim 60 \mathrm{~nm})$ and a narrow size distribution. $[93,94]$ The dense PEG corona surrounding the pLys/antisense ODN complex core provided a neutral shell to prevent against non-specific ionic interactions with plasma proteins and protect the antisense ODN against nuclease attack.[94]

To further improve the stability of the nucleic acid against degradation, the basic micelle system has been modified. Significant improvements in the stability of siRNA in blood plasma have been found when the pLys block is modified to enable covalent disulfide cross-linking in the micellar core.[91] The crosslinks provide a stable core in salt solutions and blood plasma, but are cleavable inside the cell where the concentration of disulfide reducing agents is high, enabling release of the siRNA. To reinforce the stability of the micelle, the siRNA can also be modified and cholesterol-conjugated siRNA (chol-siRNA) specifically has been used.[90] The cholesterol stabilizes the micelle structure through hydrophobic associations of the cholesterol groups in the core.[90]

Packaging of nucleic acids into micelles can significantly improve stability in plasma, but it also introduces difficulties in release of the nucleic acid from the endosome to the cytoplasm. Enhancing this process is essential to using PEC micelles as a delivery vehicle. One approach to promoting release takes advantage of the low $\mathrm{pH}$ of the endosomal compartment ( $\mathrm{pH}$ 5.5) and utilizes acid-labile linkages 
that cleave when exposed to this $\mathrm{pH} .[97,98,101]$ The therapeutic siRNA[97,98] or antisense ODN[101] is linked to the PEG through an acid-labile $\beta$-thiopropionate linkage and the micelle is formed through complexation with pLys. The detachment of the PEG segment in the endosomal compartment leads to the transport of the active nucleic acid from the endosome to the cytoplasm.

Another approach to improving endosomal release of a nucleic acid therapeutic is selection of a polypeptide cation that disrupts the endosomal membrane. For delivery of mRNA, a pH responsive peptide, pAsp(DET) was complexed with mRNA for delivery into the central nervous system.[95] A similar approach was used for delivery of siRNA, where a known pH-responsive, cationic fusogenic peptide, KALA, was used as the polycation for complexation to a PEGsiRNA copolymer.[99] In this case, the siRNA was also attached to the PEG via a disulfide linkage that is cleaved in the endosome environment, combining two methods for improving release of the therapeutic to the cytoplasm. Another study utilizes KALA, but also modifies the block copolymer structure to improve the stability of the micelle. Choi et al. utilized a 6 arm PEG-based star polymer and attached siRNA to the arms as a method for increasing charge density. When mixed with the cationic KALA, which was modified with cysteine residues for crosslinking, micelles were formed with enhanced delivery efficiency.[100]

Known membrane disruptors can also be designed to be $\mathrm{pH}$ responsive using a protection approach. For delivery of siRNA, Meyer et al. modified the amines of a melittin polypeptide with dimethylmaleic anhydride (DMMAn) to minimize their membrane disruption activity at the extracellular neutral $\mathrm{pH}$. When exposed to the 
acidic $\mathrm{pH}$ in the endosome, the DMMAn protecting groups were removed and the melittin polypeptide was able to lyse the membrane and release the siRNA therapeutic.[92]

In addition to serving to protect the nucleic acid therapeutic from nonspecific interactions with plasma proteins and enzymatic degradation, the structure of the PEC micelle is also conducive to incorporation of molecules to target specific cells. Oishi et al. fixed a lactose group, a specific ligand for recognition by asialoglycoprotein (ASGP) receptors on hepatocytes, to the surface of the micelle by attaching it to the end of the PEG block. $[97,98,101]$ This greatly enhanced the performance of the target therapeutics, both siRNA and antisense ODNs. Other targeting peptides for specific cell types have also been shown to greatly improve nucleic acid therapeutic performance. These include REKA (Arginine-Glutamic AcidLysine-Alanine) and VHPKQHR (Valine-Histidine-Proline-Lysine-GlutamineHistidine-Arginine) for targeting fibrin and endothelial cells, respectively, to deliver inhibitors against dys-regulated microRNA for the treatment of atherosclerosis.[32] This is of particular importance for atherosclerosis treatments, as it has previously proven difficult to deliver therapeutics specifically to the diseased arteries, but by using VHPKQHR, which targets a cell adhesion molecule that is more prevalent in unhealthy endothelial cells, the inhibitors against dys-regulated microRNA can be delivered specifically to diseased arteries.[32]

An additional advantage of the PEC micellar delivery system for nucleic acid therapeutics is the size of the micelles (typically in the tens of nanometers), which is of similar magnitude as natural delivery vectors such as viruses and 
lipoproteins.[93] Though the micelle size can be controlled to some degree by adjusting the PEG/polyelectrolyte ratio and concentrations, more precise control over nanoparticle size can be obtained by combining PEC units with gold nanoparticles. Kim et al demonstrated that unimer PECs composed of a single PEGpLys molecule and a single siRNA molecule can be conjugated onto gold nanoparticles through thiol-gold coordinate bonding, resulting in extremely precise size control of the delivery vehicles.[102] Interestingly, in a subsequent investigation, RNA strand rigidity was found to influence the formation of a unimer versus a PEC micelle.[103] Hayahsi et al. found that double stranded RNA formed unimer PEC complexes, whereas single stranded RNA was able to form a PEC micelle.[103] Micellar Complexes of Polypeptides and Protein

Like bulk PECs, nanoscale micellar complexes have been formed by combining polypeptides with biological proteins or synthetic polymers. Recent progress regarding applied enzymology has increased knowledge regarding structure and function of clinically-relevant enzymes, stimulating directed research toward modulating enzyme release using appropriate carrier systems. [104-106] In an effort to assess whether entrapment within nanoscale micellar complexes can maintain and preserve the enzymatic activity of lysozyme, Harada and colleagues prepared PEC micelles from a mixture of the enzyme and the anion PEG-pAsp. $[104,107]$ Lysozyme was chosen because of its availability, well-characterized structural and biological properties, and its $\mathrm{pI}(\sim 11)$ which is high enough to complex with an anionic block ionomer. The lysozyme structure remained the same 
as free lysozyme upon micelle core-incorporation, confirmed by circular dichroism, and a stable, spherical, core-shell structure was maintained without precipitation even after 1 month. However, the study did not include investigations into the coacervate or precipitate nature of the PEC core.[107]

Enzymatic activity of lysozyme was measured by assessing the hydrolysis of the $\beta$-1,4-glycosidic bond between $N$-acetylmuramic acid and $N$-acetylglucosamine. Notably, within PEC micelles, enzymatic activity increased compared to free lysozyme, mainly attributed to a decrease in the Michaelis constant (K) and was correlated with the thickness of the corona, suggesting that the corona layer could act as a reservoir for enzyme substrates. Interestingly, when nonstoichometric mixing ratios were studied, the aggregation number of PEG-pAsp and the corona thickness was found to increase with increasing PEG-pAsp; however, the aggregation number of lysozyme and the core size remained constant, independent of the mixing ratio.[106] Together, the authors conclude that these results indicate enzyme activity within the core can be tuned through the rational design of the corona thickness, which can serve as a useful tool for diagnostic and therapeutic carrier systems.[104] Micellar Complexes of Polypeptides and Synthetic Polymers

In addition to proteins, PEC micelles can result from the electrostatic complexation between polypeptides and polymers, whether synthetic or biological. The properties of the core can also be properly tuned for developing new structuring agents in the field of mesoporous inorganic materials or as new nanoparticles for effective drug release. [108-111] Warnant and colleagues took on 
this endeavor by investigating the physicochemical properties of PEC micelles formed by complexation of a double hydrophilic block copolymer (DHBC), poly(acrylic acid)- $b$-poly(acrylate methoxy poly(ethylene oxide)) (PAA- $b$-PAMPE0), and a series of five polyamines of varying $\mathrm{pKa}$ (pLys, linear and branched polyethyleneimine(PEI), poly-2-vinylpyridine(P2VP), and polyallylamine (PAH). $\mathrm{pH}$ ranges of PEC micelle formation were dependent on the $\mathrm{pKa}$ of the polyamine and all cores were found to be hydrophilic, as confirmed by the inability to incorporate pyrene. In contrast, the behavior of the cationic fluorescent molecule, Auramine 0 (AO), in the PEC micellar core was dependent on the nature of the polyamine. The local environment for AO is constrained within PEC micelles formed using P2VP, pLys and branched PEI. However, movements were less impeded in linear PEI, with PAH providing an intermediate value.[108] Nanostructures, determined by SAXS, showed that the interface between the core and corona was much less abrupt in the case of linear PEI compared to polyamine-based PEC micelles. Therefore, the corecorona nanostructures with a well-defined and dense core presented more constrained and viscous local environments, which could relate to differences in PEC phase behavior. Overall, the core density of $\mathrm{pH}$-sensitive micelles depends on the properties of the polyamine polyelectrolyte, providing insight into the formation of nanodevices and carriers and the inclusion of similarly-charged drugs.[108]

Lee et al. also describe pH-specific micelle carriers.[109] Constructed from poly(l-lactic acid)-(PLA, 3K)- $b$-PEG (2K)-b-polyhistidine (5K), they describe how flower-like micelles obtain $\mathrm{pH}$-sensitivity from the deformation of the micellar core due to the ionization of the polyhistidine at slightly acidic conditions. However, the 
co-presence of a pH-insensitive lipophilic PLA block in the core was found to prevent disintegration of the micelles and cause swelling and aggregation. The polarity of pyrene retained in the micelles increased as the $\mathrm{pH}$ was decreased from 7.4 to 6.6 , indicating a more hydrophilic environment within the micelles. The size also increased with decreasing $\mathrm{pH}$. Interestingly, the subsequent decline of pyrene polarity below pH 6.6 suggested re-self-assembly of the block copolymers, most likely forming a PLA block core with polyhistidine block relocation to the surface. Collectively, these pH-dependent physical changes of the PLA-b-PEG-b-polyhistidine micelles provide a mechanism for triggering drug release from the micelles within a small $\mathrm{pH}$ window.

Charged biopolymers, such as polysaccharides, can also enable complexation with oppositely-charged molecules for PEC micellization. [110,111] Characterization studies regarding complexes derived from pLys and hyaluronic acid-block-PEG (HA-b-PEG) of different HA lengths and PEG blocks showed a minimal molecular weight of the HA block $(9 \mathrm{kDa})$ is necessary for complexation, while a minimal molecular weight of the PEG block (5 $\mathrm{kDa}$ ) is needed to prevent macroscopic aggregation. Moreover, swelling was induced upon the addition of $\mathrm{NaCl}$ up to a critical salt concentration, forming soft "nanogel" particles, which can be further crosslinked to result in well-defined nanoassemblies. Kudo and colleagues also utilize HA, as well as chondroitin sulfate (CS), with PEG- $b$ polyarginine(pArg).[111] An added benefit to PEC micelles containing pArg is the effective arginine carrier for therapeutic applications related to nitric oxide (i.e. 
directing apoptosis, vasodilation, etc.), which is generated from inducible nitric oxide synthase in macrophages using L-arginine as a substrate.

Micellar Complexes containing Polypeptides and Other Functional Molecules

By incorporating dendrimer zinc porphyrins with polypeptides, several studies report advances of PEC micelles for cancer therapy, specifically for photodynamic treatment. [112-117] Photodynamic therapy (PDT) involves systemic administration of porphyrin or phthalocyanine-based photosensitizers (PSs), followed by local photoirradiation of solid tumors with a specific wavelength light. Nanocarriers encapsulating PSs generally have large $\pi$-conjugation domains, easily forming aggregates due to their $\pi-\pi$ and hydrophobic interactions, decreasing the generation of reactive oxygen species, or ROS, which leads to photochemical destruction of tumor vessels and tissues. To solve this issue, Nishiyama report on ionic dendrimer photosensitizers based on micelles prepared through electrostatic interaction between the anionic DPc and PEG-pLys block copolymer.[113] The DPcencapsulated PEC micelle (DPc/m) displayed 100-fold higher in vitro photocytotoxicity against HeLa cells vs. free DPc.[112] Moreover, human lung adenocarcinoma were photoirradiated and cell death induced in vitro, and antitumor properties confirmed in a mouse subcutaneous tumor model with minimal phototoxicity. Katakura and colleagues also incorporated retroviral vectors to PEG-pLys and report on increased viral gene transduction efficiency 3-to 7- fold with cancer cell lines without increasing cytotoxicity.[118] These strategies display 
potential, effectiveness, and safety of PEC micelles for future cancer therapeutic applications.

\section{Unilamellae Polymersomes}

In addition to bulk and micelle PECs, other supramolecular structures can be formed including unilamellae polymersomes and gels by mixing oppositely-charged block copolymers composed of PEG and polypeptides in aqueous medium. [119123] Polymersomes formed in this manner have been named "PICsome," a vesicle consisting of a PEC membrane sandwiched between outer and inner PEG shell layers. At this point, the PEC membrane has not been extensively characterized and could be either a coacervate of precipitate. In addition to micrometer-sized particles (1-3 $\mu \mathrm{m})$, nanosomes, or "Nano-PICsomes" can be formed and controlled in a range between 100-400 nm with membrane thickness of approximately 10-15 nm (Figure

5) by simply tuning the concentration of the ionomers.[119] Using carbodiimide crosslinker chemistry (EDC), stability and circulation properties in the bloodstream, membrane permeability and drug release properties can all be easily modified in an economically efficient manner for biomedical applications. The PICsomes have also been shown to display protease resistance[120] and $\mathrm{pH}$ sensitivity. A recent review regarding PICsomes for biomedical applications assesses recent progress.[124]

\section{Conclusion}


Polypeptides provide unique opportunities to control the phase behavior and stability of PECs in both bulk and nanoscale materials. Due to the ability to carefully control sequence and charge spacing, they serve as excellent model systems to analyze PEC phenomena. Future work using polypeptides to form PEC complexes will likely investigate the effect of sequence specific polyelectrolytes. These polyelectrolytes can resemble proteins containing intrinsically disordered regions involved in the formation of membraneless organelles that have been shown to have properties similar to coacervates, like RNA granules. $[125,126]$ This sequence specific approach will enable the investigation of orthogonal molecular interactions on PEC formation, such as $\pi-\pi$ or cation- $\pi$ interactions similar to what has been observed by introducing hydrogen bonding to PEC formation.[28] We believe that these future studies will not only shed light on these biological systems, but also provide additional understanding into what drives coacervate versus precipitate formation when simply mixing oppositely charged polymers. This will allow the creation of a versatile platform for developing self-assembled materials based on multivalent interactions that can lead to more dynamic assemblies. Additionally, this sequence specific approach will also benefit the investigation of polypeptides complexed with nucleic acids, which resemble naturally occurring macromolecular complexes found intracellularly.[127] Most of the work surveyed using polypeptide nucleic acid complexes involves little characterization of the phase behavior of the PEC complex formed. We believe that more careful characterization of polyplexes will only strengthen their applications in gene delivery and that sequence specificity 
in both the polypeptide and nucleic acids may play a role in what determines coacervate versus precipitate formation.

This review has shown that block copolymer polypeptides provide benefits for the delivery of nucleic acids, proteins, and other charged therapeutics by stabilizing the complex at the nanoscale, resembling viral vectors and enabling the addition of targeting ligands. Furthermore, polypeptide sequences can contain specific amino acids that can enable crosslinking of the complex (using cysteine), targeting capabilities, or $\mathrm{pH}$ sensitivity (using histidine) in a biocompatible manner. Notably absent from the characterization of many PEC micelles is whether the PEC core is a coacervate or precipitate, which may influence many physiochemical properties and could be pursued in the future via direct comparison to bulk PECs formed using the same polymers. It remains to be seen if the creation of solid core versus liquid core PEC micelles will affect stability in vivo, cellular internalization mechanisms, and transfection efficiency. Next generation polypeptide based PEC micelles will likely also benefit from sequence specificity that can control between a coacervate or precipitate core utilizing multivalent interactions. Additionally, PEC based delivery vehicles could benefit from the addition of more robust targeting elements, like antibodies which have been placed on the surface of hydrophobically driven polymeric micelles.[128] Overall, polypeptide based PECs and PEC micelles show great promise for the development of new materials that can shed light on biological systems and also be used as delivery vehicles, adhesives, or tissue engineering constructs.

\section{Acknowledgements}


This work was supported by the U.S. Department of Energy Office of Science,

Program in Basic Energy Sciences, Materials Sciences and Engineering Division. The authors would like to acknowledge the financial support from the National Heart, Lung, and Blood Institute (NHLBI), K99HL124279, granted to EJC. There are no conflicts of interests.

\section{References}

[1] Wang Q, Schlenoff JB. The Polyelectrolyte Complex/Coacervate Continuum. Macromolecules 2014;47:3108-16. doi:10.1021/ma500500q.

[2] Voets IK, de Keizer A, Stuart MAC. Complex coacervate core micelles. Adv Colloid Interface Sci 2009;147-148:300-18. doi:10.1016/j.cis.2008.09.012.

[3] Hammond PT. Recent explorations in electrostatic multilayer thin film assembly. Curr Opin Colloid Interface Sci 2000;4:430-42. doi:10.1016/S1359-0294(00)00022-4.

[4] Borges J, Mano JF. Molecular Interactions Driving the Layer-by-Layer Assembly of Multilayers. Chem Rev 2014;114:8883-942. doi:10.1021/cr400531v.

[5] Spruijt E, Bakker HE, Kodger TE, Sprakel J, Cohen Stuart MA, van der Gucht J. Reversible assembly of oppositely charged hairy colloids in water. Soft Matter 2011;7:8281-10. doi:10.1039/c1sm05881a.

[6] Kizilay E, Kayitmazer AB, Dubin PL. Complexation and coacervation of polyelectrolytes with oppositely charged colloids. Adv Colloid Interface Sci 2011;167:24-37. doi:10.1016/j.cis.2011.06.006.

[7] Szilagyi I, Trefalt G, Tiraferri A, Maroni P, Borkovec M. Polyelectrolyte adsorption, interparticle forces, and colloidal aggregation. Soft Matter 2014;10:2479-502. doi:10.1039/c3sm52132j.

[8] Langevin D. Complexation of oppositely charged polyelectrolytes and surfactants in aqueous solutions. A review. Adv Colloid Interface Sci 2009;147-148:170-7. doi:10.1016/j.cis.2008.08.013.

[9] Gucht JVD, Spruijt E, Lemmers M, Cohen Stuart MA. Polyelectrolyte complexes: Bulk phases and colloidal systems. J Colloid Interface Sci 2011;361:407-22. doi:10.1016/j.jcis.2011.05.080.

[10] Priftis D, Laugel N, Tirrell M. Thermodynamic Characterization of Polypeptide Complex Coacervation. Langmuir 2012;28:15947-57. doi:10.1021/la302729r.

[11] Schaaf P, Schlenoff JB. Saloplastics: Processing Compact Polyelectrolyte Complexes. Advanced Materials 2015;27:2420-32. doi:10.1002/adma.201500176.

[12] Priftis D, Tirrell M. Phase behaviour and complex coacervation of aqueous 
polypeptide solutions. Soft Matter 2012;8:9396-405.

doi:10.1039/c2sm25604e.

[13] Spruijt E, Sprakel J, Cohen Stuart MA, van der Gucht J. Interfacial tension between a complex coacervate phase and its coexisting aqueous phase.

Soft Matter 2010;6:172-8. doi:10.1039/b911541b.

[14] Priftis D, Farina R, Tirrell M. Interfacial Energy of Polypeptide Complex Coacervates Measured via Capillary Adhesion†. Langmuir 2012;28:87219. doi:10.1021/la300769d.

[15] Qin J, Priftis D, Farina R, Perry SL, Leon L, Whitmer J, et al. Interfacial Tension of Polyelectrolyte Complex Coacervate Phases. ACS Macro Lett 2014;3:565-8. doi:10.1021/mz500190w.

[16] Madene A, Jacquot M, Scher J, Desobry S. Flavour encapsulation and controlled release - a review. Int J Food Sci Tech 2006;41:1-21. doi:10.1111/j.1365-2621.2005.00980.x.

[17] Stewart RJ, Wang CS, Shao H. Advances in Colloid and Interface Science. Adv Colloid Interface Sci 2011;167:85-93. doi:10.1016/j.cis.2010.10.009.

[18] Keating CD. Aqueous phase separation as a possible route to compartmentalization of biological molecules. Acc Chem Res 2012;45:2114-24. doi:10.1021/ar200294y.

[19] Oparin A. The Origin of Primary Colloidal Systems. The Origin of Life, 1953, pp. 137-62.

[20] Koga S, Williams DS, Perriman AW, Mann S. Peptide-nucleotide microdroplets as a step towards a membrane-free protocell model. Nature Chem 2011;3:720-4. doi:doi:10.1038/nchem.1110.

[21] Song JK, Kang HC, Kim KS, Chin I-J. Microcapsules by Complex Coacervation for Electronic Ink. Mol Cryst Liq Cryst 2007;464:263/[845]-269/[851]. doi:10.1080/15421400601031199.

[22] Sun Y, Peng C, Wang X, Wang R, Chen Y, Zhang D. Phase Behavior of Polyelectrolyte Complexes and Rheological Behavior of Alumina suspensions for Direct Ink Writing. J Am Ceram Soc 2016:n/a-n/a. doi:10.1111/jace.14155.

[23] Gratson GM, Lewis JA. Phase behavior and rheological properties of polyelectrolyte inks for direct-write assembly. Langmuir 2005;21:457-64. doi:10.1021/la048228d.

[24] Kataoka K, Harada A, Nagasaki Y. Block copolymer micelles for drug delivery: Design, characterization and biological significance. Adv Drug Deliv Rev 2012;64:37-48. doi:10.1016/j.addr.2012.09.013.

[25] Pergushov DV, Müller AH, Schacher FH. Micellar interpolyelectrolyte complexes. Chem Soc Rev 2012;41:6888-901. doi:10.1039/c2cs35135h.

[26] de Kruif CG, Weinbreck F, de Vries R. Complex coacervation of proteins and anionic polysaccharides. Curr Opin Colloid Interface Sci 2004;9:340-9. doi:10.1016/j.cocis.2004.09.006.

[27] Fischer D, Li Y, Ahlemeyer B, Krieglstein J, Kissel T. In vitro cytotoxicity testing of polycations: influence of polymer structure on cell viability and hemolysis. Biomaterials 2003;24:1121-31. doi:10.1016/S0142-

9612(02)00445-3. 
[28] Perry SL, Leon L, Hoffmann KQ, Kade MJ, Priftis D, Black KA, et al. Chiralityselected phase behaviour in ionicpolypeptide complexes. Nat Comms 2015;6:1-8. doi:10.1038/ncomms7052.

[29] Priftis D, Leon L, Song Z, Perry SL, Margossian KO, Tropnikova A, et al. SelfAssembly of $\alpha$-Helical Polypeptides Driven by Complex Coacervation. Angewandte Chemie 2015;127:11280-4. doi:10.1002/ange.201504861.

[30] Amblard M, Fehrentz J-A, Martinez J, Subra G. Methods and protocols of modern solid phase peptide synthesis. Mol Biotechnol 2006;33:239-54.

[31] Fields GB, Noble RL. Solid phase peptide synthesis utilizing 9fluorenylmethoxycarbonyl amino acids. Int J Pep Prot Res 1990;35:161214. doi:10.1111/j.1399-3011.1990.tb00939.x.

[32] Kuo C-H, Leon L, Chung EJ, Huang R-T, Sontag TJ, Reardon CA, et al. Inhibition of atherosclerosis-promoting microRNAs via targeted polyelectrolyte complex micelles. J Mater Chem B 2014;2:8142-53. doi:10.1039/c4tb00977k.

[33] Cheng J, Deming TJ. Synthesis of Polypeptides by Ring-Opening Polymerization of $\alpha$-Amino Acid N-Carboxyanhydrides. Topics in Current Chemistry, vol. 310, Berlin, Heidelberg: Springer Berlin Heidelberg; 2011, pp. 1-26. doi:10.1007/128_2011_173.

[34] Perry S, Li Y, Priftis D, Leon L, Tirrell M. The Effect of Salt on the Complex Coacervation of Vinyl Polyelectrolytes. Polymers 2014;6:1756-72. doi:10.3390/polym6061756.

[35] Zhao M, Zhou J, Su C, Niu L, Liang D. Complexation behavior of oppositely charged polyelectrolytes: Effect of charge distribution. J Chem Phys 2015;142:204902. doi:10.1063/1.4921652.

[36] Zhang L, Lipik V, Miserez A. Complex Coacervates of Oppositely Charged Co-Polypeptides Inspired by the Sandcastle Worm Glue. J Mater Chem B 2016. doi:10.1039/C5TB02298C.

[37] Hoffmann KQ, Perry SL, Leon L, Priftis D, Tirrell M, de Pablo JJ. Soft Matter. Soft Matter 2015;00:1-14. doi:10.1039/C4SM02336F.

[38] Lu H, Wang J, Bai Y, Lang JW, Liu S, Lin Y, et al. Ionic polypeptides with unusual helical stability. Nat Comms 2011;2:206.

doi:10.1038/ncomms1209.

[39] Zheng C, Niu L, Pan W, Zhou J, Lv H, Cheng J, et al. Long-Term Kinetics of DNA interacting with polycations. Polymer 2014;55:2464-71. doi:10.1016/j.polymer.2014.03.038.

[40] Lee Y, Kataoka K. Delivery of Nucleic Acid Drugs. Adv Polym Sci 2012;249:95-134. doi:10.1007/12_2011_129.

[41] Soliman M, Nasanit R, Abulateefeh SR, Allen S, Davies MC, Briggs SS, et al. Multicomponent Synthetic Polymers with Viral-Mimetic Chemistry for Nucleic Acid Delivery. Mol Pharmaceutics 2012;9:1-13. doi:10.1021/mp200108q.

[42] Parelkar SS, Chan-Seng D, Emrick T. Biomaterials. Biomaterials 2011;32:2432-44. doi:10.1016/j.biomaterials.2010.12.004.

[43] Kabanov AV, Kabanov VA. DNA complexes with polycations for the delivery of genetic material into cells. Bioconjugate Chem 1995;6:7-20. 
doi:10.1021/bc00031a002.

[44] Gebhart CL, Kabanov AV. Evaluation of polyplexes as gene transfer agents. J Control Release 2001;73:401-16. doi:10.1016/S0168-3659(01)00357-1.

[45] Mann A, Richa R, Ganguli M. DNA condensation by poly-l-lysine at the single molecule level: Role of DNA concentration and polymer length. J Control Release 2008;125:252-62. doi:10.1016/j.jconrel.2007.10.019.

[46] Mann A, Thakur G, Shukla V, Ganguli M. Peptides in DNA delivery: current insights and future directions. Drug Discov Today 2008;13:152-60. doi:10.1016/j.drudis.2007.11.008.

[47] Su C, Zhao M, Zhu Z, Zhou J, Wen H, Yin Y, et al. Effect of Peptide Charge Distribution on the Structure and Kinetics of DNA Complex. Macromolecules 2015;48:756-63. doi:10.1021/ma501901b.

[48] Lai E, van Zanten JH. Monitoring DNA/Poly-L-Lysine Polyplex Formation with Time-Resolved Multiangle Laser Light Scattering. Biophysical Journal 2001;80:864-73. doi:10.1016/S0006-3495(01)76065-1.

[49] Mintzer MA, Simanek EE. Nonviral Vectors for Gene Delivery. Chem Rev 2009;109:259-302. doi:10.1021/cr800409e.

[50] Oupický D, Reschel T, Koňák Č, Oupická L. Temperature-Controlled Behavior of Self-Assembly Gene Delivery Vectors Based on Complexes of DNA with Poly( l-lysine)- graft-poly( N-isopropylacrylamide). Macromolecules 2003;36:6863-72. doi:10.1021/ma034039a.

[51] Benns JM, Choi J-S, Mahato RI, Park J-S, Kim SW. pH-Sensitive Cationic Polymer Gene Delivery Vehicle: N-Ac-poly( 1-histidine)-graft-poly( 1lysine) Comb Shaped Polymer. Bioconjugate Chem 2000;11:637-45. doi:10.1021/bc0000177.

[52] Choi YH, Liu F, Kim JS, Choi YK, Park JS, Kim SW. Polyethylene glycolgrafted poly-L-lysine as polymeric gene carrier. J Control Release 1998;54:39-48. doi:10.1016/S0168-3659(97)00174-0.

[53] Sanjoh M, Miyata K, Christie RJ, Ishii T, Maeda Y, Pittella F, et al. Dual Environment-Responsive Polyplex Carriers for Enhanced Intracellular Delivery of Plasmid DNA. Biomacromolecules 2012;13:3641-9. doi:10.1021/bm301095a.

[54] Jeong JH, Park TG, Kim SH. Self-Assembled and Nanostructured siRNA Delivery Systems. Pharm Res 2011;28:2072-85. doi:10.1007/s11095-0110412-y.

[55] Takemoto H, Ishii A, Miyata K, Nakanishi M, Oba M, Ishii T, et al. Polyion complex stability and gene silencing efficiency with a siRNA-grafted polymer delivery system. Biomaterials 2010;31:8097-105. doi:10.1016/j.biomaterials.2010.07.015.

[56] Latinwo F, Schroeder CM. Model systems for single molecule polymer dynamics. Soft Matter 2011;7:7907-13. doi:10.1039/c1sm05298e.

[57] Stevenson M, Ramos-Perez V, Singh S, Soliman M, Preece JA, Briggs SS, et al. Delivery of siRNA mediated by histidine-containing reducible polycations. J Control Release 2008;130:46-56. doi:10.1016/j.jconrel.2008.05.014.

[58] Zheng C, Niu L, Yan J, Liu J, Luo Y, Liang D. Structure and stability of the 
complex formed by oligonucleotides. Phys Chem Chem Phys 2012;14:7352-9. doi:10.1039/c2cp24086f.

[59] Black KA, Priftis D, Perry SL, Yip J, Byun WY, Tirrell M. Protein Encapsulation via Polypeptide Complex Coacervation. ACS Macro Lett 2014;3:1088-91. doi:10.1021/mz500529v.

[60] Girod S, Boissière M, Longchambon K, Begu S, Tourne-Pétheil C, Devoisselle JM. Polyelectrolyte complex formation between iotacarrageenan and poly(l-lysine) in dilute aqueous solutions: a spectroscopic and conformational study. Carbohydrate Polymers 2004;55:37-45. doi:10.1016/j.carbpol.2003.07.006.

[61] Ortony JH, Hwang DS, Franck JM, Waite JH, Han S. Asymmetric Collapse in Biomimetic Complex Coacervates Revealed by Local Polymer and Water Dynamics. Biomacromolecules 2013;14:1395-402. doi:10.1021/bm4000579.

[62] Xia J, Dubin PL. Protein-Polyelectrolyte Complexes. In: Dubin P, Bock J, Davis R, Schulz DN, Thies C, editors. Macromolecular Complexes in Chemistry and Biology, Berlin, Heidelberg: Springer Berlin Heidelberg; 1994, pp. 247-71. doi:10.1007/978-3-642-78469-9_15.

[63] Thu B, Bruheim P, Espevik T, Smidsrød O, Soon-Shiong P, Skjåk-Bræk G. Alginate polycation microcapsules. Biomaterials 1996;17:1031-40. doi:10.1016/0142-9612(96)84680-1.

[64] Bartkowiak A, Hunkeler D. Carrageenan-oligochitosan microcapsules: optimization of the formation process. Colloids Surf, B 2001;21:285-98.

[65] Wang J, Liu C, Wei J, Chi P, Lu X, Yin M. Synthesis and properties of chitosan/polypeptide bioconjugate composite. Biomed Mater 2007;2:32. doi:10.1088/1748-6041/2/1/006.

[66] Harada A, Kataoka K. Formation of polyion complex micelles in an aqueous milieu from a pair of oppositely-charged block copolymers with poly (ethylene glycol) segments. Macromolecules 1995;28:5294-9.

[67] Harada A, Kataoka K. Chain Length Recognition: Core-Shell Supramolecular Assembly from Oppositely Charged Block Copolymers. Science 1999;283:65-7. doi:10.1126/science.283.5398.65.

[68] Harada A, Kataoka K. Formation of Stable and Monodispersive Polyion Complex Micelles in Aqueous Medium from Poly(L-lysine) And Poly(Ethylene Glycol)-Poly(Aspartic Acid) Block Copolymer. J of Macromolecular Sc, Part A 1997;34:2119-33. doi:10.1080/10601329708010329.

[69] Harada A, Kataoka K. Effect of Charged Segment Length on Physicochemical Properties of Core-Shell Type Polyion Complex Micelles from Block Ionomers. Macromolecules 2003;36:4995-5001. doi:10.1021/ma025737i.

[70] Wibowo A, Osada K, Matsuda H, Anraku Y, Hirose H, Kishimura A, et al. Morphology Control in Water of Polyion Complex Nanoarchitectures of Double-Hydrophilic Charged Block Copolymers through Composition Tuning and Thermal Treatment. Macromolecules 2014;47:3086-92. doi:10.1021/ma500314d. 
[71] Mutaf OF, Kishimura A, Mochida Y, Kim A, Kataoka K. Induction of Secondary Structure through Micellization of an Oppositely Charged Pair of Homochiral Block-and Homopolypeptides in an Aqueous Medium. Macromol Rapid Commun 2015;36:1958-64. doi:10.1002/marc.201500368.

[72] Kakizawa Y, Harada A, Kataoka K. Environment-Sensitive Stabilization of Core-Shell Structured Polyion Complex Micelle by Reversible CrossLinking of the Core through Disulfide Bond. J Am Chem Soc 1999;121:11247-8. doi:10.1021/ja993057y.

[73] Park J-S, Akiyama Y, Yamasaki Y, Kataoka K. Preparation and Characterization of Polyion Complex Micelles with a Novel Thermosensitive Poly(2-isopropyl-2-oxazoline) Shell via the Complexation of Oppositely Charged Block Ionomers. Langmuir 2007;23:138-46. doi:10.1021/la061431j.

[74] Ren J, Zhang Y, Zhang J, Gao H, Liu G, Ma R, et al. pH/Sugar Dual Responsive Core-Cross-Linked PIC Micelles for Enhanced Intracellular Protein Delivery. Biomacromolecules 2013:130924081120002. doi:10.1021/bm4007387.

[75] Putnam D, Zelikin AN, Izumrudov VA, Langer R. Polyhistidine-PEG:DNA nanocomposites for gene delivery. Biomaterials 2003;24:4425-33. doi:10.1016/S0142-9612(03)00341-7.

[76] Wolfert MA, Schacht EH, Toncheva V, Ulbrich K, Nazarova O, Seymour LW. Characterization of Vectors for Gene Therapy Formed by Self-Assembly of DNA with Synthetic Block Co-Polymers. Hum Gene Ther 1996;7:2123-33. doi:10.1089/hum.1996.7.17-2123.

[77] Katayose S, Kataoka K. Water-soluble polyion complex associates of DNA and poly (ethylene glycol)-poly (L-lysine) block copolymer. Bioconjugate Chem 1997;8:702-7.

[78] Katayose S, Kataoka K. Remarkable increase in nuclease resistance of plasmid DNA through supramolecular assembly with poly(ethylene glycol)?poly(L-lysine) block copolymer. J Pharm Sci 1998;87:160-3. doi:10.1021/js970304s.

[79] Harada-Shiba M, Yamauchi K, Harada A, Takamisawa I, Shimokado K, Kataoka K. Polyion complex micelles as vectors in gene therapy-pharmacokinetics and in vivo gene transfer. Gene Ther 2002;9:407-14.

[80] Itaka K, Yamauchi K, Harada A, Nakamura K, Kawaguchi H, Kataoka K. Polyion complex micelles from plasmid DNA and poly(ethylene glycol)poly(l-lysine) block copolymer as serum-tolerable polyplex system: physicochemical properties of micelles relevant to gene transfection efficiency. Biomaterials 2003;24:4495-506. doi:10.1016/S01429612(03)00347-8.

[81] Osada K, Oshima H, Kobayashi D, Doi M, Enoki M, Yamasaki Y, et al. Quantized Folding of Plasmid DNA Condensed with Block Catiomer into Characteristic Rod Structures Promoting Transgene Efficacy. J Am Chem Soc 2010;132:12343-8. doi:10.1021/ja102739b.

[82] Tockary TA, Osada K, Chen Q, Machitani K, Dirisala A, Uchida S, et al. 
Tethered PEG Crowdedness Determining Shape and Blood Circulation Profile of Polyplex Micelle Gene Carriers. Macromolecules 2013;46:658592. doi:10.1021/ma401093z.

[83] Bikram M, Ahn C-H, Chae SY, Lee M, Yockman JW, Kim SW. Biodegradable Poly(ethylene glycol)- co-poly( l-lysine)- g-histidine Multiblock

Copolymers for Nonviral Gene Delivery. Macromolecules 2004;37:190316. doi:10.1021/ma035650c.

[84] Ziady A, Gedeon C, Muhammad O, Stillwell V, Oette S, Fink T, et al. Minimal toxicity of stabilized compacted DNA nanoparticles in the murine lung. Mol Ther 2003;8:948-56. doi:10.1016/j.ymthe.2003.09.002.

[85] Boylan NJ, Kim AJ, Suk JS, Adstamongkonkul P, Simons BW, Lai SK, et al. Enhancement of airway gene transfer by DNA nanoparticles using a pHresponsive block copolymer of polyethylene glycol and poly-l-lysine. Biomaterials 2012;33:2361-71. doi:10.1016/j.biomaterials.2011.11.080.

[86] Itaka K, Ohba S, Miyata K, Kawaguchi H, Nakamura K, Takato T, et al. Bone Regeneration by Regulated In Vivo Gene Transfer Using Biocompatible Polyplex Nanomicelles. Mol Ther 2007;15:1655-62.

doi:10.1038/sj.mt.6300218.

[87] Miyata K, Oba M, Nakanishi M, Fukushima S, Yamasaki Y, Koyama H, et al. Polyplexes from Poly(aspartamide) Bearing 1,2-Diaminoethane Side Chains Induce pH-Selective, Endosomal Membrane Destabilization with Amplified Transfection and Negligible Cytotoxicity. J Am Chem Soc 2008;130:16287-94. doi:10.1021/ja804561g.

[88] Li Y, Osada K, Chen Q, Tockary TA, Dirisala A, Takeda KM, et al. Toroidal Packaging of pDNA into Block Ionomer Micelles Exerting Promoted in vivo Gene Expression. Biomacromolecules 2015;16:2664-71. doi:10.1021/acs.biomac.5b00491.

[89] Nomoto T, Fukushima S, Kumagai M, Machitani K, Arnida, Matsumoto Y, et al. Three-layered polyplex micelle as a multifunctional nanocarrier platform for light-induced systemic gene transfer. Nat Comms 2014;5:110. doi:10.1038/ncomms4545.

[90] Oe Y, Christie RJ, Naito M, Low SA, Fukushima S, Toh K, et al. Biomaterials. Biomaterials 2014:1-9. doi:10.1016/j.biomaterials.2014.05.041.

[91] Christie RJ, Miyata K, Matsumoto Y, Nomoto T, Menasco D, Lai TC, et al. Effect of Polymer Structure on Micelles Formed between siRNA and Cationic Block Copolymer Comprising Thiols and Amidines. Biomacromolecules 2011;12:3174-85. doi:10.1021/bm2006714.

[92] Meyer M, Philipp A, Oskuee R, Schmidt C, Wagner E. Breathing Life into Polycations: Functionalization with pH-Responsive Endosomolytic Peptides and Polyethylene Glycol Enables siRNA Delivery. J Am Chem Soc 2008;130:3272-3. doi:10.1021/ja710344v.

[93] Kataoka K, Togawa H, Harada A, Yasugi K, Matsumoto T, Katayose S. Spontaneous formation of polyion complex micelles with narrow distribution from antisense oligonucleotide and cationic block copolymer in physiological saline. Macromolecules 1996;29:8556-7.

[94] Harada A, Togawa H, Kataoka K. Physicochemical properties and nuclease 
resistance of antisense-oligodeoxynucleotides entrapped in the core of polyion complex micelles composed of poly (ethylene glycol)-poly (llysine) block copolymers. Eur J Pharm Sci 2001;13:35-42.

[95] Uchida S, Itaka K, Uchida H, Hayakawa K, Ogata T, Ishii T, et al. In Vivo Messenger RNA Introduction into the Central Nervous System Using Polyplex Nanomicelle. PLoS ONE 2013;8.

doi:10.1371/journal.pone.0056220.

[96] Baba M, Itaka K, Kondo K, Yamasoba T, Kataoka K. Journal of Controlled Release. J Control Release 2015;201:41-8.

doi:10.1016/j.jconrel.2015.01.017.

[97] Oishi M, Nagasaki Y, Itaka K, Nishiyama N, Kataoka K. Lactosylated Poly(ethylene glycol)-siRNA Conjugate through Acid-Labile $\beta$ Thiopropionate Linkage to Construct $\mathrm{pH}$-Sensitive Polyion Complex Micelles Achieving Enhanced Gene Silencing in Hepatoma Cells. J Am Chem Soc 2005;127:1624-5. doi:10.1021/ja044941d.

[98] Oishi M, Nagasaki Y, Nishiyama N, Itaka K, Takagi M, Shimamoto A, et al. Enhanced Growth Inhibition of Hepatic Multicellular Tumor Spheroids by Lactosylated Poly(ethylene glycol)-siRNA Conjugate Formulated in PEGylated Polyplexes. ChemMedChem 2007;2:1290-7. doi:10.1002/cmdc.200700076.

[99] Lee SH, Kim SH, Park TG. Intracellular siRNA delivery system using polyelectrolyte complex micelles prepared from VEGF siRNA-PEG conjugate and cationic fusogenic peptide. Biochem Biophys Res Commun 2007;357:511-6. doi:10.1016/j.bbrc.2007.03.185.

[100] Choi SW, Lee SH, Mok H, Park TG. Multifunctional siRNA delivery system: Polyelectrolyte complex micelles of six-arm PEG conjugate of siRNA and cell penetrating peptide with crosslinked fusogenic peptide. Biotechnol Progress 2009;26:NA-NA. doi:10.1002/btpr.310.

[101] Oishi M, Nagatsugi F, Sasaki S, Nagasaki Y, Kataoka K. Smart Polyion Complex Micelles for Targeted Intracellular Delivery of PEGylated Antisense Oligonucleotides Containing Acid-Labile Linkages. ChemBioChem 2005;6:718-25. doi:10.1002/cbic.200400334.

[102] Kim HJ, Takemoto H, Yi Y, Zheng M, Maeda Y, Chaya H, et al. Precise Engineering of siRNA Delivery Vehicles to Tumors Using Polyion Complexes and Gold Nanoparticles. ACS Nano 2014;8:8979-91. doi:10.1021/nn502125h.

[103] Hayashi K, Chaya H, Fukushima S, Watanabe S, Takemoto H, Osada K, et al. Influence of RNA Strand Rigidity on Polyion Complex Formation with Block Catiomers. Macromol Rapid Commun 2016;37:486-93. doi:10.1002/marc.201500661.

[104] Harada A, Kataoka K. Pronounced activity of enzymes through the incorporation into the core of polyion complex micelles made from charged block copolymers. J Control Release 2001;72:85-91. doi:10.1016/S0168-3659(01)00264-4.

[105] Harada A, Kataoka K. On-Off Control of Enzymatic Activity Synchronizing with Reversible Formation of Supramolecular Assembly from Enzyme and 
Charged Block Copolymers. J Am Chem Soc 1999;121:9241-2.

doi:10.1021/ja9919175.

[106] Harada A, Kataoka K. Novel Polyion Complex Micelles Entrapping Enzyme Molecules in the Core. 2. Characterization of the Micelles Prepared at Nonstoichiometric Mixing Ratios †. Langmuir 1999;15:4208-12. doi:10.1021/la981087t.

[107] Harada A, Kataoka K. Novel polyion complex micelles entrapping enzyme molecules in the core: Preparation of narrowly-distributed micelles from lysozyme and poly(ethylene glycol)-poly(aspartic acid) block copolymer in aqueous medium. Macromolecules 1998;31:288-94.

[108] Warnant J, Marcotte N, Reboul J, Layrac G, Aqil A, Jerôme C, et al. Physicochemical properties of $\mathrm{pH}$-controlled polyion complex (PIC) micelles of poly(acrylic acid)-based double hydrophilic block copolymers and various polyamines. Anal Bioanal Chem 2012;403:1395-404. doi:10.1007/s00216-012-5947-1.

[109] Lee ES, Oh KT, Kim D, Youn YS, Bae YH. Tumor pH-responsive flower-like micelles of poly(l-lactic acid)-b-poly(ethylene glycol)-b-poly(l-histidine). J Control Release 2007;123:19-26. doi:10.1016/j.jconrel.2007.08.006.

[110] Novoa-Carballal R, Pergushov DV, Müller AH. Interpolyelectrolyte complexes based on hyaluronic acid-block-poly (ethylene glycol) and polyL-lysine. Soft Matter 2013;9:4297-303. doi:10.1039/c3sm27549c.

[111] Kudo S, Nagasaki Y. Facile and Quantitative Synthesis of a Poly(ethylene glycol)- b-Poly( (l-arginine) Block Copolymer and Its Use for the Preparation of Polyion Complex Micelles with Polyanions for Biomedical Applications. Macromol Rapid Commun 2015;36:1916-22. doi:10.1002/marc.201500224.

[112] Jang W-D, Nakagishi Y, Nishiyama N, Kawauchi S, Morimoto Y, Kikuchi M, et al. Polyion complex micelles for photodynamic therapy: Incorporation of dendritic photosensitizer excitable at long wavelength relevant to improved tissue-penetrating property. J Control Release 2006;113:73-9. doi:10.1016/j.jconrel.2006.03.009.

[113] Nishiyama N, Nakagishi Y, Morimoto Y, Lai P-S, Miyazaki K, Urano K, et al. Enhanced photodynamic cancer treatment by supramolecular nanocarriers charged with dendrimer phthalocyanine. J Control Release 2009;133:245-51. doi:10.1016/j.jconrel.2008.10.010.

[114] Nishiyama N, Stapert HR, Zhang G-D, Takasu D, Jiang D-L, Nagano T, et al. Light-Harvesting Ionic Dendrimer Porphyrins as New Photosensitizers for Photodynamic Therapy. Bioconjugate Chem 2003;14:58-66. doi:10.1021/bc025597h.

[115] Stapert HR, Nishiyama N, Jiang D-L, Aida T, Kataoka K. Polyion Complex Micelles Encapsulating Light-Harvesting Ionic Dendrimer Zinc Porphyrins. Langmuir 2000;16:8182-8. doi:10.1021/la000423e.

[116] Zhang GD, Harada A, Nishiyama N, Jiang DL. Polyion complex micelles entrapping cationic dendrimer porphyrin: effective photosensitizer for photodynamic therapy of cancer. J Control Release 2003;93:141-50. doi:10.1016/j.jconrel.2003.05.002. 
[117] Zhang G-D, Nishiyama N, Harada A, Jiang D-L, Aida T, Kataoka K. pHsensitive Assembly of Light-Harvesting Dendrimer Zinc Porphyrin Bearing Peripheral Groups of Primary Amine with Poly(ethylene glycol)- bpoly(aspartic acid) in Aqueous Solution. Macromolecules 2003;36:1304-9. doi:10.1021/ma025735y.

[118] Katakura H, Harada A, Kataoka K, Furusho M, Tanaka F, Wada H, et al. Improvement of retroviral vectors by coating with poly(ethylene glycol)poly(L-lysine) block copolymer(PEG-PLL). J Gene Med 2004;6:471-7. doi:10.1002/jgm.519.

[119] Anraku Y, Kishimura A, Oba M, Yamasaki Y, Kataoka K. Spontaneous formation of nanosized unilamellar polyion complex vesicles with tunable size and properties. J Am Chem Soc 2010;132:1631-6.

doi:10.1021/ja908350e.

[120] Kishimura A, Koide A, Osada K, Yamasaki Y, Kataoka K. Encapsulation of Myoglobin in PEGylated Polyion Complex Vesicles Made from a Pair of Oppositely Charged Block Ionomers: A Physiologically Available Oxygen Carrier. Angew Chem Int Ed 2007;46:6085-8.

doi:10.1002/anie.200701776.

[121] Koide A. Semipermeable Polymer Vesicle (PICsome) Self-Assembled in Aqueous Medium from a Pair of Oppositely Charged Block Copolymers: Physiologically Stable Micro-/Nanocontainers of WaterSoluble Macromolecules. J Am Chem Soc 2006;128:5988-9. doi:10.1021/ja057993r.

[122] Oana H, Morinaga M, Kishimura A, Kataoka K. Direct formation of giant unilamellar vesicles from microparticles of polyion complexes and investigation of their properties using a microfluidic chamber. Soft Matter 2013;9:5448-58. doi:10.1039/C3SM00089C.

[123] Pham TTH, Wang J, Werten M, Snijkers F, de Wolf F, Stuart MAC, et al. Multi-responsive physical gels formed by a biosynthetic asymmetric triblock protein polymer and a polyanion. Soft Matter 2013;9:8923-30. doi:10.1039/c3sm51751a.

[124] Kishimura A. Development of polyion complex vesicles (PICsomes) from block copolymers for biomedical applications. Polym J 2013;45:892-7. doi:10.1038/pj.2013.33.

[125] Brangwynne CP, Eckmann CR, Courson DS, Rybarska A, Hoege C, Gharakhani J, et al. Germline P Granules Are Liquid Droplets That Localize by Controlled Dissolution/Condensation. Science 2009;324:1729-32. doi:10.1126/science.1172046.

[126] Weber SC, Brangwynne CP. Getting RNA and Protein in Phase. Cell 2012;149:1188-91. doi:10.1016/j.cell.2012.05.022.

[127] Lin Y, Protter DSW, Rosen MK, Parker R. Formation and Maturation of Phase-Separated Liquid Droplets by RNA-Binding Proteins. Molecular Cell 2015;60:208-19. doi:10.1016/j.molcel.2015.08.018.

[128] Chan DPY, Owen SC, Shoichet MS. Double Click: Dual Functionalized Polymeric Micelles with Antibodies and Peptides. Bioconjugate Chem 2013;24:105-13. doi:10.1021/bc300511a. 


\section{Figure Captions}

Figure 1- Solid and liquid polyelectrolyte complexes using pLys and pGlu. Solid complexes (left image) are characterized by their amorphous shape are formed using L-pLys(PLK) and L-pGlu(PLE). Coacervates are formed using L-pLys (PLK) and racemic pGlu(P(D,L)E) and are characterized by their spherical shape. Scale bar 25 microns. FTIR spectra of the amide one region of both complexes are shown below images. Both complexes have a peak around 1642-1646 $\mathrm{cm}^{-1}$ indicative of a random coil configuration, and another peak at another peak at $1563 \mathrm{~cm}^{-1}$ corresponding to the glutamic acid side chain. Only solid complexes have peaks at 1610 and $1678 \mathrm{~cm}^{-1}$ indicative of $\beta$-strand structure. Reproduced from Hoffmann et al. with permission from the Royal Society of Chemistry. [37]

Figure 2-Schematic illustration for preparation and intracellular trafficking of cross-linked polyplexes with $\mathrm{pH}$ and disulfide reduction dual intracellular environment-sensitivity for improved gene delivery. Reprinted with permission from Sanjoh et al. Copyright 2012 American Chemical Society.

Figure 3-Preparation of polypeptide complex coacervates with encapsulated proteins. Reproduced with permission from Black et al.[59]

Figure 4- TEM images of PICs at $25 \mathrm{C}$ and $1 \mathrm{mg} / \mathrm{mL}$ (scale bar $100 \mathrm{~nm}$ ) with PEG fraction of (a) $12.1 \%$ (b) $11.1 \%$ and (c)10.0\%. Image reproduced with permission from Wibowo et al.[70]

Figure 5. Cryogenic phase-contrast transmission electron microscopic image of Nano-PICsomes after crosslinking obtained at a total polymer concentration of 
$1 \mathrm{mg} \mathrm{ml}^{-1}$. Arrow indicates vesicle walls. Image reproduced with permission from Anraku et al.[119] 


\section{Figures}

\section{Figure 1}

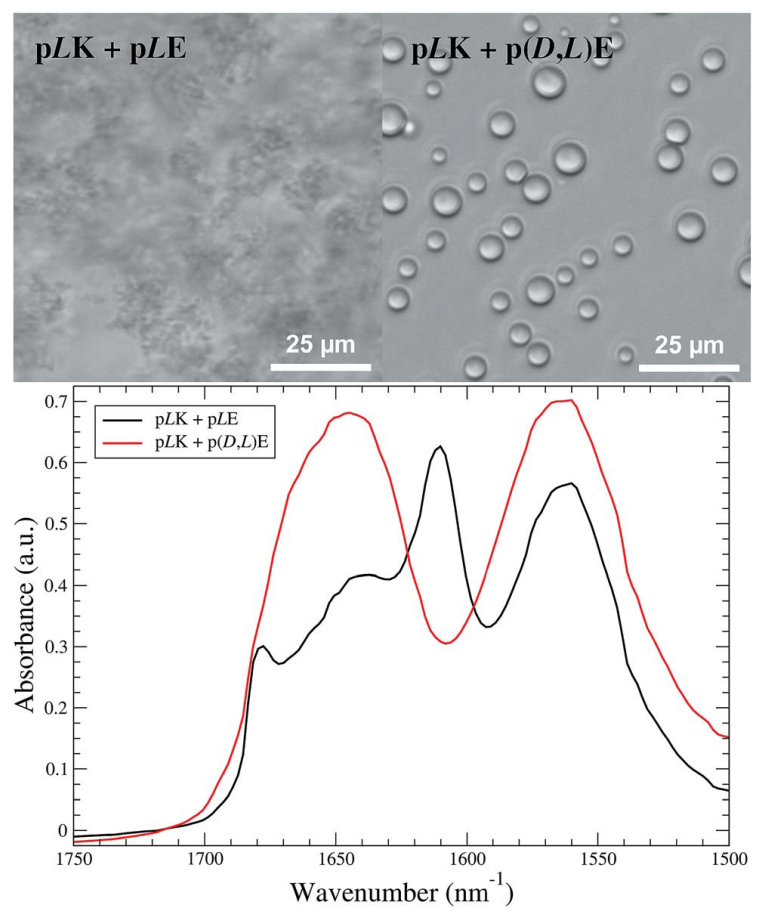

Figure 2

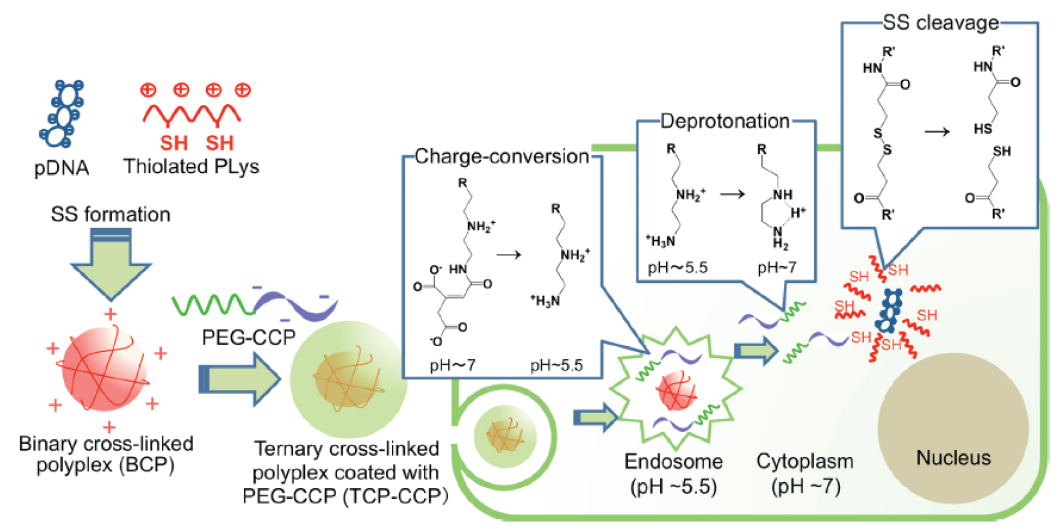

\section{Figure 3:}

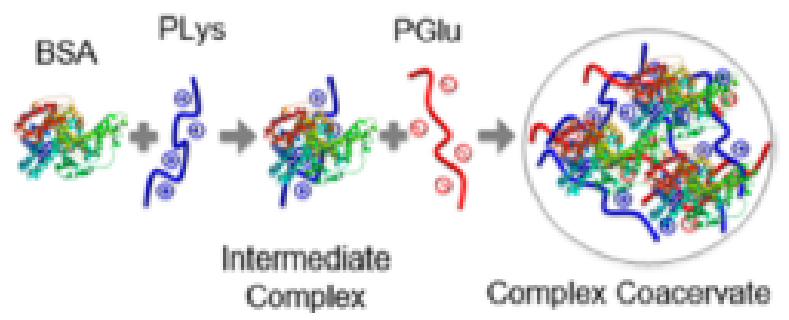




\section{Figure 4}
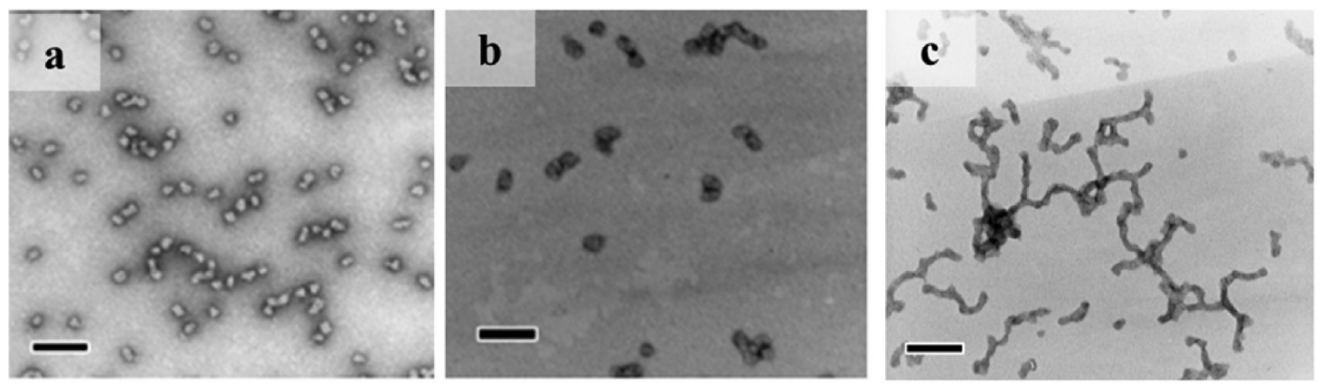

Figure 5

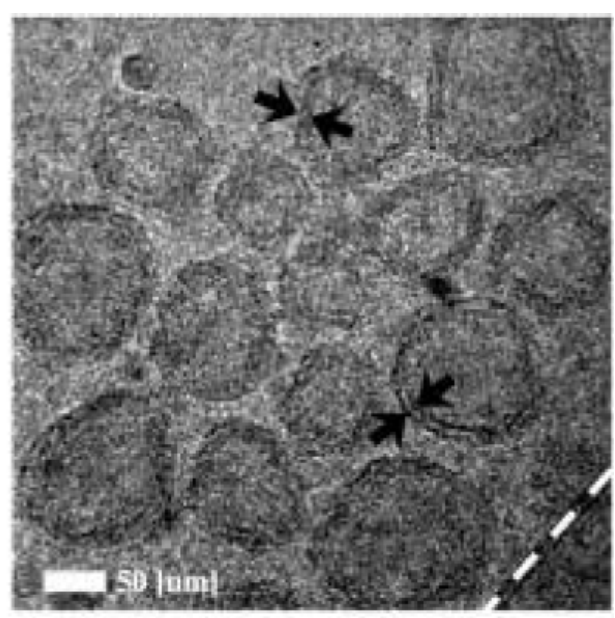

